\title{
The RASputin effect
}

\author{
Benjamin Boettner and Linda Van Aelst ${ }^{\mathbf{1}}$ \\ Cold Spring Harbor Laboratory, Cold Spring Harbor, New York 11724, USA
}

By 1916, Rasputin had become an important figure of the corruption of the Romanov court and its treacherous, unpatriotic behavior. For many people on the fringes of the court, Rasputin's corruption was taken as the cause of all of Russia's problems itself. There was the belief that if one could get rid of Rasputin the revolution may not happen. . . . In a sense, Rasputin's assassination in December 1916, should be seen as just one of a number of palace coup plots.

\section{Orlando Figes \\ Cambridge University}

Cells committed to malignancy are subject to a complex series of events prior to reaching their full tumorigenic potential within a given type of cancer. These events are often distinguished by the expression of particular physiological characteristics. The development of a colorectal tumor is probably the best investigated paradigm. Initially, an epithelial cell is driven out of its normal state to give rise to dysplastic aberrant crypt foci. Further genetic events ensue that lead to the advancement through various adenomas, each showing a specific appearance, until a carcinoma arises that ultimately metastasizes (Kinzler and Vogelstein 1996). More generally, tumors originating from different cell types have individual etiologies that may follow a specific path along definable stages. What will be of broad relevance, however, is the acquisition of a set of features that Hanahan and Weinberg (2000) recently defined as "the hallmarks of cancer." A successful tumor needs to perform the following tasks: it has to (1) attain independence from growth signals, (2) become insensitive to growth inhibitory mechanisms, (3) escape the apoptotic machinery that detects imbalances in cellular behavior, (4) acquire a limitless replicative potential, (5) model its own angiogenic support system, and (6) eventually switch to an invasive and metastatic physiology (Hanahan and Weinberg 2000). The ability of a tumor to corrupt the normal cellular circuitries that underlie these aspects depends on distinct genetic alterations accumulating in a single cancer cell.

The Ras oncogenes N-RAS, H-RAS, and K-RAS are mutated in $30 \%$ of human cancers (Bos 1989), and their principle function has been ascribed to point (1) of the

${ }^{1}$ Corresponding author.

E-MAIL vanaelst@cshl.org; FAX (516) 367-8815.

Article and publication are at http://www.genesdev.org/cgi/doi/10.1101/ gad.1020602. above aspects, namely, the conveyance of growth factor autonomy to a tumor cell. It is commonly believed that Ras proteins in this context propagate a signal through the Raf/MEK/MAPK cassette to the nucleus to manipulate the cell cycle machinery. In addition, work performed in numerous laboratories over the past decade has also revealed a potential participation of Ras in other aspects of tumorigenicity. To give some illustrative examples, another of Ras's activities, namely, stimulation of the PI3-kinase/AKT pathway, is thought to exert an anti-apoptotic effect (acquired trait 3; Downward 1998). Furthermore, oncogenic Ras has been implicated in the control of vascular endothelial growth factor (VEGF), and interfering with the Ras/Raf/MAPK pathway in endothelial cells disrupts neovascularization in vivo (acquired trait 5; Rak et al. 1995; Eliceiri et al. 1998). Finally, metastatic behavior can be stimulated greatly by oncogenic Ras (acquired trait 6; Chambers and Tuck 1993; Webb et al. 1998). Thus, although cancer cells in many cases may use more dominant mechanisms to counteract apoptosis, provoke angiogenesis, and metastasize, Ras oncoproteins may provide an ancillary input. These principles have been worked out primarily in mouse systems, and most of them are yet to be reevaluated in human cancers, which in the light of the findings described by Hamad et al. (2002) in this issue will attain further relevance.

\section{Ras effect(ors)}

Experimental evidence for the oncogenic potential of Ras has been obtained mostly by classical focus-forming assays performed in rodent cells, such as NIH3T3 cells, and by injection of rodent cells that have been engineered to express an oncogenic transgene into immunocompromised mice. Whereas the former assays assess anchorage independence under serum-starved conditions, the latter informs about the ability to form a solid tumor in vivo. Rodent fibroblasts like NIH3T3 cells are immortalized and can be transformed by forced expression of oncogenic Ras proteins relatively efficiently. The generation of mutant Ras variants that harbor mutations in their effector loop region (amino acids 32-40) has been instrumental in the further dissection of the effector pathways that are required for Ras's transforming activity in rodent cells (White et al. 1995; Rodriguez-Viciana et al. 1997). This sequence of nine amino acids is highly conserved among oncogenic Ras proteins and interacts with immediate binding partners that, in turn, specify 
downstream signaling. The three best investigated candidate effectors are the Raf-kinases that activate the MAPK module, the p110 catalytic subunit of PI3-kinase, a generator of the second messenger phosphatidylinositol 3,4,5-phosphate $\left(\mathrm{PIP}_{3}\right)$, and RalGEF isoforms that function as guanine nucleotide exchange factors for the small Ral GTPase (Fig. 1). In mutant terms, a V12 mutation in Ras is combined with an additional mutation residing in the effector loop region that dictates binding specificity. An H-Ras ${ }^{\mathrm{V} 12 / \mathrm{S} 35}$ protein binds exclusively to Raf-kinases but not to PI3-kinase or RalGEFs; H$\mathrm{Ras}^{\mathrm{V} 12 / \mathrm{G} 37}$ selects for RalGEF without binding to Raf or PI3-kinase, and H-Ras ${ }^{\mathrm{V} 12 / \mathrm{C} 40}$ only shows affinity for PI3kinase (White et al. 1995; Khosravi-Far et al. 1996; Rodriguez-Viciana et al. 1997). The introduction of these double-mutant cDNAs into NIH3T3 cells unveiled a scenario in which the Raf/MEK/MAPK cascade acts as the principle pathway to confer oncogenic potential to fibroblasts. However, additional important insights were revealed in these studies. Although a Raf-specific Ras mutant, indeed, was transforming, and this activity could be mimicked by activated forms of Raf, such as the artificially membrane-tethered Raf-CAAX mutant, both transforming potentials were weaker compared with the original H-Ras ${ }^{\mathrm{V} 12}$ mutant (Khosravi-Far et al. 1996). These attenuated phenotypes could be reinforced significantly by simultaneous expression of constitutively active PI3-kinase or RalGEF variants (Urano et al. 1996; White et al. 1996; Rodriguez-Viciana et al. 1997). In contrast, dominant-negative mutants of PI3-kinase or dominant-negative Ral protein can diminish the focus-forming activity of H-Ras ${ }^{\text {V12 }}$ (Urano et al. 1996; RodriguezViciana et al. 1997). Together, this and other experimental evidence has led to a conceptual framework in which Raf-induced signaling via MAPK transmits the key transforming signal emanating from Ras, but the facilitating effects of other Ras-induced pathways are required concurrently.

\section{Ras takes different routes in the transformation of mouse and human cells}

When comparing the range of human cancers with mutations in one of the Ras (N-RAS, $\mathrm{H}-R A S$, and $\mathrm{K}-R A S)$ genes with the tumor types arising in mice that have been genetically engineered to produce constitutively active forms of Ras, a number of discrepancies surface. This is not a phenomenon unique to Ras, but can be considered a recurrent theme intrinsic to a number of such interspecies comparisons. Mice lacking functional copies of tumor suppressor genes, such as $p 53, N F-1$, and $R b$, are prone to develop incompletely overlapping sets of tumors compared with human cancer patients carrying analogous mutations (Donehower et al. 1992; Jacks et al. 1992, 1994). RAS mutations in human subjects occur at high frequency in tumors derived from colon, pancreatic, lung, and thyroid tissues (Bos 1989). Whereas the importance of mutational K-ras activation for the onset of lung cancer has been elegantly shown in mice (Johnson et al. 2001), tumors in pancreatic, colon, or thyroid environments have not been observed in mice carrying an oncogenic copy of ras. In contrast, rodents show a preponderance of breast and skin cancers in response to carcinogens (Zarbl et al. 1985; Quintanilla et al. 1986; Miyamoto et al. 1990), whereas human breast cancers very rarely result from $R A S$ mutations.

Prompted by these inconsistencies, Hamad and her coworkers (2002) asked whether the different patterns in tumor formation in mice versus humans could be caused by a difference in effector usage by Ras, and they unearthed some surprising results. The authors based their experiments on an important previous finding by Hahn and colleagues (1999). Until recently, the transformation of human cells in a research environment has been impossible because, unlike mouse cells, they are subject to replicative senescence (see acquired trait 4 above). Experimentally, introduction of oncogenic RAS into primary cells rapidly drives them into premature cellular senescence (Serrano et al. 1997), thwarting any further analysis. Many tumors breach the senescence barrier by up-regulating the catalytically active component of telomerase, hTERT (Bryan and Cech 1999), and Hahn and coworkers (1999) were able to successfully transform human cells by simultaneous expression of hTERT, the early region of the simian virus 40, and oncogenic RAS. In their present paper, Hamad and her colleagues (2002) used the same tools to examine the H-Ras effector loop mutants described above for their transforming effects in
Figure 1. Ras-dependent effector pathways in mouse and human involved in tumorigenesis. (Left) Mouse paradigm is depicted. The activation of Raf (bolt arrow) by oncogenic Ras presents the major tumorigenic effect, whereas PI3-kinase- and RalGDS-controlled pathways facilitate transformation. In human cells (right) the activation of RalGEF appears to be the principal transformation pathway, although PI3-kinase and Raf-dependent signaling is required. Other potential Ras targets that so far have not been examined well enough are contained in the box.

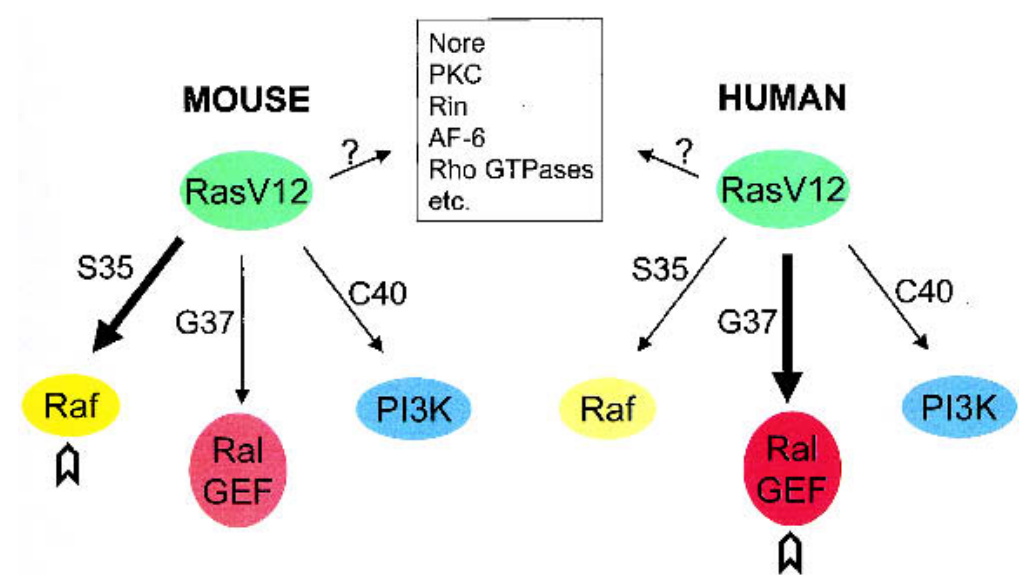


human primary fibroblasts, primary human embryonic kidney (HEK) epithelial cells, and human astrocytes. Surprisingly, in a carefully controlled series of experiments, the retrovirally introduced RalGEF-specific H-Ras ${ }^{\mathrm{V} 12 / \mathrm{G} 37}$ mutant gave rise to transformation, but the Raf- and PI3kinase-specific $\mathrm{H}-\mathrm{Ras}^{\mathrm{V} 12 / \mathrm{S} 35}$ and $\mathrm{H}-\mathrm{Ras}^{\mathrm{V} 12 / \mathrm{C} 40}$ mutants were virtually ineffective (Fig. 1). Therefore, constitutively activated $\mathrm{H}$-Ras relays a transformation-essential signal to RalGEF as tested in soft agar assays. In contrast, activation of Raf or PI3-kinase was not sufficient to bring about a transforming effect in human cells. Further experimentation convincingly corroborated these findings. Neither an activated form of Raf, $\Delta$ Raf1-22W, nor a constitutively active PI3-kinase subunit, p110-CAAX, was able to transform human cells, individually or cooperatively. On the other hand, mere expression of a constitutively active RalGEF could master transformation of HEK cells, and the introduction of a dominant-negative RalA mutant, RalA ${ }^{\mathrm{N} 28}$, suppressed transformation by H-Ras ${ }^{\mathrm{V} 12 / \mathrm{G} 37}$. Thus, activation of the RalGEF effector branch by RAS appears to be a necessary requirement for the transformation of human cells. However, Hamad and her colleagues observed that activated Raf and PI3-kinase mutants enhanced the $\mathrm{H}-\mathrm{Ras}^{\mathrm{V} 12 / \mathrm{G} 37}$ oncogenic effect. This is even more clearly reflected in experiments performed in immuno-compromised mice inoculated with the Ras-manipulated human HEK cells. In this more complex system, the H-Ras ${ }^{\mathrm{V} 12 / \mathrm{G} 37}$ mutant on its own did not give rise to tumor formation, and neither did the other effector loop variants. Concurrent expression of all three mutants, in contrast, was highly tumorigenic, but omission of the H-Ras ${ }^{\mathrm{V} 12 / \mathrm{G} 37}$ mutant eliminates tumorigenicity.

Taken together, these data disclose fundamental differences in the mechanistics with which Ras may signal in mouse and human tumor cells. Their observations led the authors to hypothesize a model in which RalGEF activation downstream of Ras is essential for its oncogenic potential in human cancers, and they suggest that the cooperation of Raf and PI3-kinase effector pathways are required to elicit tumor growth in vivo (Fig. 1).

\section{How could RalGEFs promote tumor formation downstream of Ras?}

Given that MAPK provides the major proliferative signal in Ras-transformed mouse NIH3T3 cells, the proliferative activity of RalGEF in human cells necessitates an alternative cell cycle stimulatory pathway. Indeed, Hamad et al. (2002) excluded any MAPK elevating activity of the H-Ras ${ }^{\mathrm{V} 12 / \mathrm{G} 37}$ mutant in human cells. To date, there is sparse evidence for an impact of Ral GTPases on the cell cycle machinery. Earlier, Gille and Downward (1999) described a positive effect of an activated mutant form of a RalGEF family member, Rlf-CAAX, on cyclin D1 transcription and E2F activity, two principal agents of $\mathrm{G}_{1} / \mathrm{S}$ progression in human cells (Gille and Downward 1999; Wolthuis and Bos 1999). This was corroborated by Henry et al. (2000), who reported that activated Ral can elevate the transcription level of cyclin D1 as well. Ral appears to impinge on cyclin D1 transcription in an NFкB-dependent fashion by activating the cytoplasmically dormant factor that then, in turn, binds to an NF-кBspecific element in the cyclin D1 promotor (Henry et al. 2000). Also, MAPK activation by Ras in various systems triggers the cyclin D1 promotor but does so in a different way (Cheng et al. 1998). These potential mechanisms have been determined in rodent fibroblasts, and there is a strong need to reevaluate them in human cells affected by Ras. It is noteworthy that Yamazaki et al. (2001) performed experiments in human fibrosarcoma (HT1080) cells that can grow anchorage-independently in soft agar owing to an activating mutation in their $\mathrm{N}-R A S$ gene. Dominant-negative Ral could interfere with this phenotype. Furthermore, once adhesion of HT1080 cells was abrogated constitutively, activated Ral appeared to decrease the levels of $\mathrm{p} 27^{\mathrm{Kip} 1}$, an inhibitor of cell cyclepromoting cyclin/CDK complexes, whereas the opposite effect was observed with dominant-negative Ral (Yamazaki et al. 2001). Of interest, de Ruiter and colleagues (2001) showed that in the context of the human DLD1 colon carcinoma cell line, a Ras/Ral-specific pathway leads to the phosphorylation of the forkhead transcription factor AFX at two critical residues. This modification represses the transcriptional activity of the latter to decrease the level of the $\mathrm{p} 27^{\mathrm{Kip} 1}$ cell cycle inhibitor, consequently alleviating G1 progression of the cell cycle (de Ruiter et al. 2001). p2 $7^{\text {Kip1 }}$ degradation was also found to be facilitated by Rho GTPase-dependent signaling, which is also thought to be active downstream of oncogenic Ras (Weber et al. 1997; Hu et al. 1999). Thus, disabling $\mathrm{p} 27^{\mathrm{Kip} 1}$ may be a function of several pathways that are governed by Ras.

Apart from cell cycle-directed effects, several other cellular activities of Ral are beginning to emerge. Ral, by virtue of its binding partner RalBP1/RLIP76, modulates the internalization of growth factor-dependent receptors, such as epidermal growth factor (EGF) receptor and insulin receptor (Nakashima et al. 1999; Jullien-Flores et al. 2000). Both constitutively active and dominant-negative mutants of Ral efficiently block EGF uptake in human A431 epithelial carcinoma and other cells (Nakashima et al. 1999). This is suggestive of the notion that Ral needs to cycle between its GTP- and GDP-bound forms under normal homeostatic circumstances and that its overactivation by oncogenic Ras could shift the balance to allow sustained activity of EGF receptors. Interestingly, A431 cells that are exposed to EGF are induced to proliferate and to show metastasis-associated properties. Interestingly, EGF-receptor engagement also has been demonstrated to stimulate Src-kinase with an impact on its own internalization (Wilde et al. 1999). More recently, Goi et al. (2000) were able to show that EGFdependent Src activation depends on Ral activity, and they expanded the spectrum of Src-substrates in this pathway by including Stat3 and cortactin to the list. Thus, it will be interesting to see whether an EGF/Ral/ Src pathway negatively feeds back to promote the uptake of EGF-receptors. Interference with growth factor receptor internalization by Ral may well be generalized 
to other cancer-relevant receptor tyrosine kinase systems in the future. Another, more recently discovered function of Ral is its association with the exocyst, a multisubunit complex that, in polarized cells like those of neuronal and epithelial lineages, governs the vectorial transport of a subset of secretory vesicles. One of its components, Sec5, has been identified as a Ral-effector (Brymora et al. 2001; Moskalenko et al. 2002). Moskalenko and his coworkers (2002) interfered with Ral activity using multiple approaches, including ectopic expression of dominant Ral mutants and RalAdirected RNAi, and they conclusively showed that endogenous Ral-regulation is required for proper exocyst function. GFP-labeled EGF receptors, for example, mislocalize when normal Ral activity is abolished (Moskalenko et al. 2002). Hence, membrane trafficking events both endocytic and exocytic in nature are critically regulated by Ral. At this point, there is no evidence that these phenomena participate in the proliferative effect of oncogenic Ras in human cells, but it will be exciting to follow the experiments that may further test a possible participation of these pathways in tumor formation.

It should be kept in mind, that although an activated RalGEF could mimic the transforming effect of H-Ras ${ }^{\mathrm{V} 12 / \mathrm{G} 37}$, it did not do so as effectively as the Ras mutant, suggesting that $\operatorname{Ras}^{\mathrm{V} 12 / \mathrm{G} 37}$ may engage other effectors to exert its full oncogenicity (Fig. 1). Certainly, it will be interesting to monitor whether this mutant can interact with other potential Ras partners such as Nore (Khokhlatchev et al. 2002), Rin (Han and Colicelli 1995), and AF-6 (Van Aelst et al. 1995), but the generated evidence for a relevant role of a RalGEF/Ral effector pathway promoting transformation in human cells is striking. In mouse cells the inhibition of a number of signaling molecules that are believed to exert a function downstream of Ras also curbs Ras's transforming potential. For example, particular functions of the Rho GTPases, namely, Rho, Rac, and Cdc42, downstream of oncogenic Ras have been found to contribute to transformation. Moreover, Rho family activators can act as oncogenes in rodent assays and have been found to be mutated in human cancers (Boettner and Van Aelst 2002). Strikingly, gene-targeted mice lacking both functional copies of the TIAM gene, which encodes a Rac activator, are resistent to Ras-induced skin tumorigenesis (Malliri et al. 2002). The means by which Rho GTPases are activated by oncogenic Ras remains an open issue, but there is mounting evidence for their contribution to cancer development in humans.

\section{Does function follow redundancy?}

The proposed principal transformation pathway emanating from Ras in human cells leads to the induction of RalGEF, which, in turn, stimulates the exchange of Ral GDP for GTP. However, this pathway will most certainly prove to be more complex. There are multiple variants of oncogenic Ras as well as of RalGEF and Ral. Differences in expression levels and timing, as well as different subcellular localizations of these isoforms, could further complicate the matter. Whereas most cancer studies, including the one by Hamad et al. (2002), have been conducted with H-RAS, it will be interesting to see whether K-RAS and N-RAS will yield the same patterns in human cells. In particular, K-RAS will be of importance, because it is the predominantly mutated RAS gene in human cancers (Ellis and Clark 2000). From a developmental point of view, gene-targeting studies in mice have revealed that it is only the loss of K-Ras function that is essential for mouse embryonic development, whereas N-Ras and H-Ras are dispensable (Johnson et al. 1997). This could be a reflection of differences in expression between different types of Ras in different tissues, with K-Ras being the most common isoform. Alternatively, K-Ras, N-Ras, and H-Ras could all be ubiquitously expressed but perform slightly distinct roles, with $\mathrm{K}$-Ras taking center stage. If the latter were true, the prevalence of mutationally activated K-Ras in cancer could mirror this dominance. There also is mounting evidence for a differential localization of Ras isoforms. Whereas H-Ras has been traced to lipid rafts, cholesterolenriched patches within the plasma membrane, K-Ras appears to be excluded from them and instead is targeted to the disordered plasma membrane (Prior and Hancock 2001). Moreover, Chiu et al. (2002) presented evidence that H-Ras and N-Ras can be activated and form productive signaling complexes in the endoplasmic reticulum and Golgi compartments. These observations clearly could have implications for effector availability, interactions, and the initiation of downstream signals. In the light of the findings by Hamad et al. (2002), in particular, expression and subcellular compartmentalization of RalGEF family members could be important.

As mentioned above, there are several RalGEF family isoforms as well (Wolthuis and Bos 1999; de Bruyn et al. 2000; Rebhun et al. 2000; Shao and Andres 2000). Members of RalGDS, Rgl, and Rlf subfamilies comprise a subgroup of Ral activators that, owing to a C-terminal Rasbinding domain (RBD), all can interact with oncogenic Ras (Wolthuis and Bos 1999). Hamad et al. (2002) have chosen Rlf for their experiments because this member of the family had shown the most pronounced Ras-association in previous studies (Esser et al. 1998), but others might fulfill an equivalent function. However, given the pleiotropy among Ras GTPases and RalGEF proteins, there may be preferential matches occurring in vivo that are of particular relevance for the development of certain tumor types.

\section{Conclusion}

In summary, the analysis of effector specification in Rasinduced tumorigenesis that Hamad and her colleagues (2002) conducted in a strictly comparative way in mouse and human cells suggests fundamental differences between the two systems. Whereas in mice Ras sends its oncogenic signal primarily through Raf, in human cells the oncogene appears to depend mainly on its RalGEF effector branch for its transforming ability. It should be 
emphasized that, at the same time, Raf- and PI3-kinase controlled pathways are additionally required for tumor formation. This is illustrated by the fact that genetic loci encoding components of both pathways have been found to be mutationally modified in tumors (Phillips et al. 1998; Cantley and Neel 1999; Hoshino et al. 1999; Ma et al. 2000). Most recently, a genome-wide cancer study has led to the identification of $B R A F$ mutations in human tumor cell lines (Davies et al. 2002). It awaits to be seen whether this or other endeavors will detect aberrant alleles or misfunction of Ral signaling elements. The biochemical investigation of the molecular connections between Ral signaling and the cell cycle machinery will become an area diligently pursued. As in the case of cancer trials that aim to intervene pharmacologically with Raf/MEK/MAPK signaling components (Stevenson et al. 1999; Cunningham et al. 2000; Sebolt-Leopold 2000), elements of the RalGEF/Ral pathway may well become attractive target molecules. However, irrespective of these questions, the present study by Hamad et al. (2002) should cause appreciation of the fact that tumor biology in humans on the mechanistic level may not always be explicable by simple extrapolation from more tractable experimental systems like the mouse.

\section{Acknowledgments}

We are indebted to Eve-Ellen Govek and Juan Mendez for critically reading the text. B.B. is a fellow of the Deutsche Akademie der Naturforscher Leopoldina. L.V.A. is supported by grants from the National Institutes of Health, the U.S. Army, and the NF Foundation Inc.

\section{References}

Boettner, B. and Van Aelst, L. 2002. The role of Rho GTPases in disease development. Gene 286: 155-174.

Bos, J.L. 1989. ras oncogenes in human cancer: A review. Cancer Res. 49: 4682-4689.

Bryan, T.M. and Cech, T.R. 1999. Telomerase and the maintenance of chromosome ends. Curr. Opin. Cell Biol. 11: 318 324.

Brymora, A., Valova, V.A., Larsen, M.R., Roufogalis, B.D., and Robinson, P.J. 2001. The brain exocyst complex interacts with RalA in a GTP-dependent manner: Identification of a novel mammalian Sec3 gene and a second Sec15 gene. J. Biol. Chem. 276: 29792-29797.

Cantley, L.C. and Neel, B.G. 1999. New insights into tumor suppression: PTEN suppresses tumor formation by restraining the phosphoinositide 3-kinase/AKT pathway. Proc. Natl. Acad. Sci. 96: 4240-4245.

Chambers, A.F. and Tuck, A.B. 1993. Ras-responsive genes and tumor metastasis. Crit. Rev. Oncog. 4: 95-114.

Cheng, M., Sexl, V., Sherr, C.J., and Roussel, M.F. 1998. Assembly of cyclin D-dependent kinase and titration of $\mathrm{p} 27^{\mathrm{Kip} 1}$ regulated by mitogen-activated protein kinase kinase (MEK1). Proc. Nat1. Acad. Sci. 95: 1091-1096.

Chiu, V.K., Bivona, T., Hach, A., Sajous, J.B., Silletti, J., Wiener, H., Johnson, R.L., Cox, A.D., and Philips, M.R. 2002. Ras signalling on the endoplasmic reticulum and the Golgi. Nat. Cell Biol. 4: 343-350.

Cunningham, C.C., Holmlund, J.T., Schiller, J.H., Geary, R.S.,
Kwoh, T.J., Dorr, A., and Nemunaitis, J. 2000. A phase I trial of c-Raf kinase antisense oligonucleotide ISIS 5132 administered as a continuous intravenous infusion in patients with advanced cancer. Clin. Cancer Res. 6: 1626-1631.

Davies, H., Bignell, G.R., Cox, C., Stephens, P., Edkins, S., Clegg, S., Teague, J., Woffendin, H., Garnett, M.J., Bottomley, W., et al. 2002. Mutations of the BRAF gene in human cancer. Nature 417: 949-954.

de Bruyn, K.M., de Rooij, J., Wolthuis, R.M., Rehmann, H., Wesenbeek, J., Cool, R.H., Wittinghofer, A.H., and Bos, J.L. 2000. RalGEF2, a pleckstrin homology domain containing guanine nucleotide exchange factor for Ral. J. Biol. Chem. 275: 29761-29766.

de Ruiter, N.D., Burgering, B.M., and Bos, J.L. 2001. Regulation of the Forkhead transcription factor AFX by Ral-dependent phosphorylation of threonines 447 and 451. Mol. Cell. Biol. 21: 8225-8235.

Donehower, L.A., Harvey, M., Slagle, B.L., McArthur, M.J., Montgomery, Jr., C.A., Butel, J.S., and Bradley, A. 1992. Mice deficient for p53 are developmentally normal but susceptible to spontaneous tumours. Nature 356: 215-221.

Downward, J. 1998. Mechanisms and consequences of activation of protein kinase B/Akt. Curr. Opin. Cell Biol. 10: 262 267.

Eliceiri, B.P., Klemke, R., Stromblad, S., and Cheresh, D.A. 1998. Integrin $\alpha v \beta 3$ requirement for sustained mitogen-activated protein kinase activity during angiogenesis. J. Cell Biol. 140: 1255-1263.

Ellis, C.A. and Clark, G. 2000. The importance of being K-Ras. Cell Signal 12: 425-434.

Esser, D., Bauer, B., Wolthuis, R.M., Wittinghofer, A., Cool, R.H., and Bayer, P. 1998. Structure determination of the Rasbinding domain of the Ral-specific guanine nucleotide exchange factor Rlf. Biochemistry 37: 13453-13462.

Gille, H. and Downward, J. 1999. Multiple ras effector pathways contribute to $\mathrm{G}_{1}$ cell cycle progression. J. Biol. Chem. 274: 22033-22040.

Goi, T., Shipitsin, M., Lu, Z., Foster, D.A., Klinz, S.G., and Feig, L.A. 2000. An EGF receptor/Ral-GTPase signaling cascade regulates c-Src activity and substrate specificity. $E M B O J$. 19: 623-630.

Hahn, W.C., Counter, C.M., Lundberg, A.S., Beijersbergen, R.L., Brooks, M.W., and Weinberg, R.A. 1999. Creation of human tumour cells with defined genetic elements. Nature 400: 464-468.

Hamad, N.M., Elconin, J.H., Karnoub, A.E., Bai, W., Rich, J.N., Abraham, R.T., Der, C.J., and Counter, C.M. 2002. Distinct requirements for Ras oncogenesis in human versus mouse cells. Genes \& Dev. (this issue).

Han, L. and Colicelli, J. 1995. A human protein selected for interference with Ras function interacts directly with Ras and competes with Raf1. Mol. Cell. Biol. 15: 1318-1323.

Hanahan, D. and Weinberg, R.A. 2000. The hallmarks of cancer. Cell 100: 57-70.

Henry, D.O., Moskalenko, S.A., Kaur, K.J., Fu, M., Pestell, R.G., Camonis, J.H., and White, M.A. 2000. Ral GTPases contribute to regulation of cyclin D1 through activation of NF-кB. Mol. Cell. Biol. 20: 8084-8092.

Hoshino, R., Chatani, Y., Yamori, T., Tsuruo, T., Oka, H., Yoshida, O., Shimada, Y., Ari-i, S., Wada, H., Fujimoto, J., et al. 1999. Constitutive activation of the $41-/ 43-\mathrm{kDa}$ mitogenactivated protein kinase signaling pathway in human tumors. Oncogene 18: 813-822.

$\mathrm{Hu}, \mathrm{W} .$, Bellone, C.J., and Baldassare, J.J. 1999. RhoA stimulates p2 $7^{\text {Kip }}$ degradation through its regulation of cyclin E/CDK2 activity. J. Biol. Chem. 274: 3396-3401. 
Jacks, T., Fazeli, A., Schmitt, E.M., Bronson, R.T., Goodell, M.A., and Weinberg, R.A. 1992. Effects of an $\mathrm{Rb}$ mutation in the mouse. Nature 359: 295-300.

Jacks, T., Shih, T.S., Schmitt, E.M., Bronson, R.T., Bernards, A., and Weinberg, R.A. 1994. Tumour predisposition in mice heterozygous for a targeted mutation in Nf1. Nat. Genet. 7: 353-361.

Johnson, L., Greenbaum, D., Cichowski, K., Mercer, K., Murphy, E., Schmitt, E., Bronson, R.T., Umanoff, H., Edelmann, W., Kucherlapati, R., et al. 1997. K-ras is an essential gene in the mouse with partial functional overlap with N-ras. Genes \& Dev. 11: 2468-2481.

Johnson, L., Mercer, K., Greenbaum, D., Bronson, R.T., Crowley, D., Tuveson, D.A., and Jacks, T. 2001. Somatic activation of the K-ras oncogene causes early onset lung cancer in mice. Nature 410: 1111-1116.

Jullien-Flores, V., Mahe, Y., Mirey, G., Leprince, C., MeunierBisceuil, B., Sorkin, A., and Camonis, J.H. 2000. RLIP76, an effector of the GTPase Ral, interacts with the AP2 complex: Involvement of the Ral pathway in receptor endocytosis. $J$. Cell Sci. 113: 2837-2844.

Khokhlatchev, A., Rabizadeh, S., Xavier, R., Nedwidek, M., Chen, T., Zhang, X., Seed, B., and Avruch, J. 2002. Identification of a novel ras-regulated proapoptotic pathway. Curr. Biol. 12: 253-265.

Khosravi-Far, R., White, M.A., Westwick, J.K., Solski, P.A., Chrzanowska-Wodnicka, M., Van Aelst, L., Wigler, M.H., and Der, C.J. 1996. Oncogenic Ras activation of Raf/mitogen-activated protein kinase-independent pathways is sufficient to cause tumorigenic transformation. Mol. Cell. Biol. 16: 3923-3933.

Kinzler, K.W. and Vogelstein, B. 1996. Lessons from hereditary colorectal cancer. Cell 87: 159-170.

Ma, Y.Y., Wei, S.J., Lin, Y.C., Lung, J.C., Chang, T.C., WhangPeng, J., Liu, J.M., Yang, D.M., Yang, W.K., and Shen, C.Y. 2000. PIK3CA as an oncogene in cervical cancer. Oncogene 19: $2739-2744$.

Malliri, A., Van Der Kammen, R.A., Clark, K., Van Der Valk, M., Michiels, F., and Collard, J.G. 2002. Mice deficient in the Rac activator Tiam1 are resistant to Ras-induced skin tumours. Nature 417: 867-871.

Miyamoto, S., Sukumar, S., Guzman, R.C., Osborn, R.C., and Nandi, S. 1990. Transforming c-Ki-ras mutation is a preneoplastic event in mouse mammary carcinogenesis induced in vitro by $N$-methyl-N-nitrosourea. Mol. Cell. Biol. 10: 1593-1599.

Moskalenko, S., Henry, D.O., Rosse, C., Mirey, G., Camonis, J.H., and White, M.A. 2002. The exocyst is a Ral effector complex. Nat. Cell Biol. 4: 66-72.

Nakashima, S., Morinaka, K., Koyama, S., Ikeda, M., Kishida, M., Okawa, K., Iwamatsu, A., Kishida, S., and Kikuchi, A. 1999. Small G protein Ral and its downstream molecules regulate endocytosis of EGF and insulin receptors. EMBO $J$. 18: $3629-3642$.

Phillips, W.A., St. Clair, F., Munday, A.D., Thomas, R.J., and Mitchell, C.A. 1998. Increased levels of phosphatidylinositol 3-kinase activity in colorectal tumors. Cancer 83: 41-47.

Prior, I.A. and Hancock, J.F. 2001. Compartmentalization of Ras proteins. J. Cell Sci. 114: 1603-1608.

Quintanilla, M., Brown, K., Ramsden, M., and Balmain, A. 1986. Carcinogen-specific mutation and amplification of Ha-ras during mouse skin carcinogenesis. Nature 322: 78-80.

Rak, J., Mitsuhashi, Y., Bayko, L., Filmus, J., Shirasawa, S., Sasazuki, T., and Kerbel, R.S. 1995. Mutant ras oncogenes upregulate VEGF/VPF expression: Implications for induction and inhibition of tumor angiogenesis. Cancer Res. 55: 45754580 .
Rebhun, J.F., Castro, A.F., and Quilliam, L.A. 2000. Identification of guanine nucleotide exchange factors (GEFs) for the Rap1 GTPase. Regulation of MR-GEF by M-Ras-GTP interaction. J. Biol. Chem. 275: 34901-34908.

Rodriguez-Viciana, P., Warne, P.H., Khwaja, A., Marte, B.M., Pappin, D., Das, P., Waterfield, M.D., Ridley, A., and Downward, J. 1997. Role of phosphoinositide 3-OH kinase in cell transformation and control of the actin cytoskeleton by Ras. Cell 89: 457-467.

Sebolt-Leopold, J.S. 2000. Development of anticancer drugs targeting the MAP kinase pathway. Oncogene 19: 6594-6599.

Serrano, M., Lin, A.W., McCurrach, M.E., Beach, D., and Lowe, S.W. 1997. Oncogenic ras provokes premature cell senescence associated with accumulation of p53 and p16INK4a. Cell 88: 593-602.

Shao, H. and Andres, D.A. 2000. A novel RalGEF-like protein, RGL3, as a candidate effector for rit and Ras. J. Biol. Chem. 275: 26914-26924.

Stevenson, J.P., Yao, K.S., Gallagher, M., Friedland, D., Mitchell, E.P., Cassella, A., Monia, B., Kwoh, T.J., Yu, R., Holmlund, J., et al. 1999. Phase I clinical/pharmacokinetic and pharmacodynamic trial of the c-raf-1 antisense oligonucleotide ISIS 5132 (CGP 69846A). I. Clin. Oncol. 17: 2227-2236.

Urano, T., Emkey, R., and Feig, L.A. 1996. Ral-GTPases mediate a distinct downstream signaling pathway from Ras that facilitates cellular transformation. EMBO J. 15: 810-816.

Van Aelst, L., White, M., and Wigler, M. 1995. Ras partners. Cold Spring Harbor Symp. Quant. Biol. 59: 181-186.

Webb, C.P., Van Aelst, L., Wigler, M.H., and Woude, G.F. 1998. Signaling pathways in Ras-mediated tumorigenicity and metastasis. Proc. Natl. Acad. Sci. 95: 8773-8778.

Weber, J.D., Hu, W., Jefcoat, Jr., S.C., Raben, D.M., and Baldassare, J.J. 1997. Ras-stimulated extracellular signal-related kinase 1 and RhoA activities coordinate platelet-derived growth factor-induced $\mathrm{G}_{1}$ progression through the independent regulation of cyclin D1 and p27. J. Biol. Chem. 272: 32966-32971.

White, M.A., Nicolette, C., Minden, A., Polverino, A., Van Aelst, L., Karin, M., and Wigler, M.H. 1995. Multiple Ras functions can contribute to mammalian cell transformation. Cell 80: 533-541.

White, M.A., Vale, T., Camonis, J.H., Schaefer, E., and Wigler, M.H. 1996. A role for the Ral guanine nucleotide dissociation stimulator in mediating Ras-induced transformation. J. Biol. Chem. 271: 16439-16442.

Wilde, A., Beattie, E.C., Lem, L., Riethof, D.A., Liu, S.H., Mobley, W.C., Soriano, P., and Brodsky, F.M. 1999. EGF receptor signaling stimulates SRC kinase phosphorylation of clathrin, influencing clathrin redistribution and EGF uptake. Cell 96: 677-687.

Wolthuis, R.M. and Bos, J.L. 1999. Ras caught in another affair: The exchange factors for Ral. Curr. Opin. Genet. Dev. 9: 112-117.

Yamazaki, Y., Kaziro, Y., and Koide, H. 2001. Ral promotes anchorage-independent growth of a human fibrosarcoma, HT1080. Biochem. Biophys. Res. Commun. 280: 868-873.

Zarbl, H., Sukumar, S., Arthur, A.V., Martin-Zanca, D., and Barbacid, M. 1985. Direct mutagenesis of Ha-ras-1 oncogenes by $N$-nitroso- $N$-methylurea during initiation of mammary carcinogenesis in rats. Nature 315: 382-385. 